\title{
Differential aspects of the disease and treatment of Thoracic Acute Aortic Dissection (TAAD) - the European experience
}

\author{
John Pepper \\ National Institute for Health Research (NIHR), Cardiovascular Biological Research unit (cBRU), Royal Brompton Hospital, London, UK \\ Correspondence to: John Pepper. Department of Cardiothoracic Surgery Royal Brompton Hospital, London, SW3 6NP, UK. Email: j.pepper@rbht.nhs.uk.
}

\begin{abstract}
The management of patients with acute aortic dissection continues to be a challenge. It is an uncommon but lethal condition which continues to be under-diagnosed and under-treated. In this review, the term acute aortic syndrome is preferred in order to embrace the closely related pathologies of intramural hematoma $(\mathrm{IMH})$ and penetrating aortic ulcer (PAU).
\end{abstract}

Keywords: Thoracic aorta; dissection; guidelines

Submitted Oct 14, 2015. Accepted for publication May 09, 2016.

doi: 10.21037 /acs.2016.06.05

View this article at: http://dx.doi.org/10.21037/acs.2016.06.05

Over the last 5 years there has been an explosion of interest and proficiency in the application of endovascular approaches to the treatment of acute type B dissection. These are well summarized in the 2014 ESC Guidelines and reflect the changes in thinking that have occurred since 2010 when the AHA/ACC guidelines were published. Diagnostic techniques have improved markedly enabling a more accurate anatomical diagnosis in more than $95 \%$ of cases.

Despite these advances, delayed referral to a cardiothoracic center is common and in many units the patient has already received anti-platelet agents when the diagnosis of an acute type A dissection is finally made. Such confusion and delay add to the morbidity and risk of operation because of the need for large quantities of blood products and their subsequent effect on the lungs. Early diagnosis can be achieved by careful history taking and clinical examination, but in the high pressure environment of a rapid access chest pain centre this is frequently curtailed. Much work is needed to find a robust point-of-care test that could act as a trigger for ordering a CT scan.

In highly selected cases, especially where there is significant co-morbidity, endovascular techniques have been applied to the treatment of type A but the number of patients is not yet sufficient to allow for validation.

The purpose of this review is to highlight a European perspective on aortic dissection and contrast it with those from other parts of the world. Within the 28 countries of the European Union there are significant differences in practice. Some of these differences are due to the preference of experienced cardiovascular units and some to the demography and geography of the country.

The incidence of aortic dissection in the general population in Europe is not accurately known. A populationbased study reported the combined incidence of aortic dissection and aneurysm (1). Between 1987 and 2002, 4,425 cases of aortic dissection were identified within a population of 8.7 million people, which equates to 3.4 per 100,000 per year. The annual incidence of aortic aneurysm and dissection combined increased by $50 \%$ in men and $30 \%$ in women during these 16 years.

The ten year results from the Oxford Vascular Study (2) based on a population of 92,728 in Oxfordshire, United Kingdom (UK), was conducted from 2002 to 2012. Among 155 patients with 174 acute events there were 52 incident events. This suggests an annual incidence of 6 per 100,000, (95\% CI: 4-7), which comprises 37 with type A dissection and 15 with type $\mathrm{B}$ within this population.

Community screening of 30,412 individuals who participated in the Malmo Diet and Cancer Survey produced reliable measures of the event rate of treated aortic disease in a European population (3). They reported rates of 15 per 100,000 patient years for aortic dissection, 9 for thoracic aortic aneurysm and 27 for abdominal aortic aneurysm. This was supported by 20 years of follow-up. 


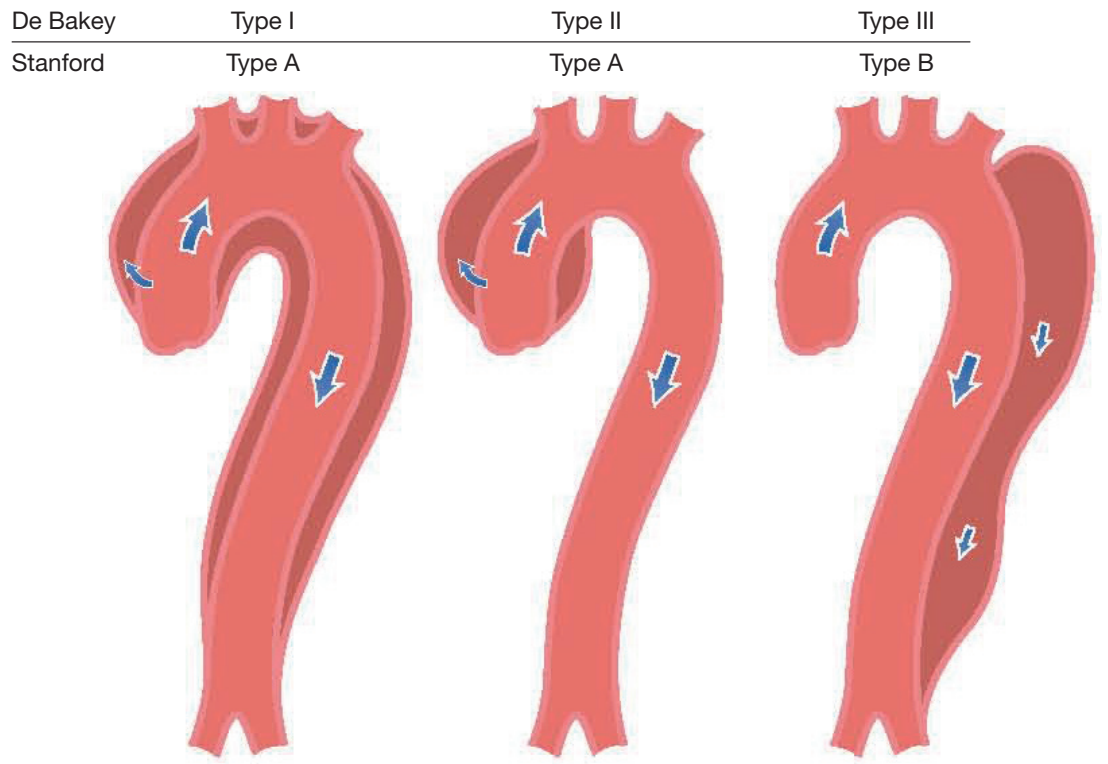

Figure 1 Classification of aortic dissection.

Unlike many previous studies, the data of patients based on community and hospital settings were kept separate. The incidence of aortic dissection appears to be increasing. This kind of data is only available in selected countries that allow access to unselected population-based medical information.

\section{Acute thoracic aortic syndrome}

In a discussion of Thoracic Acute Aortic Dissection we should take into account the whole spectrum of acute aortic syndromes (AAS) which includes aortic dissection (AD), intramural hematoma (IMH) and penetrating aortic ulcer (PAU). This is well laid out in the ESC 2014 Guidelines on the Diagnosis and Treatment of Aortic Diseases (4). The AHA Guidelines are four years older (2010) and so do not give such prominence to the treatment of IMH and PAU, nor to the need for serial imaging in those patients with initial negative imaging but a strong clinical suspicion of AAS. The Global Burden Disease 2010 project has demonstrated that the overall global death rate from aortic aneurysms and $\mathrm{AD}$ increased from 2.49 per 100,000 to 2.78 per 100,000 between 1990 and 2010 with higher rates for men (5). By contrast, the prevalence and incidence of abdominal aortic aneurysms has declined over

the last 20 years. The burden increases with age and men are more often affected than women.

Acute thoracic aortic syndromes are defined as emergency conditions with similar clinical characteristics involving the aorta. There is a common pathway for the different presentations of AAS that eventually leads to a breakdown of the intima and media. This may result in IMH, PAU or in separation of the layers of the aortic wall leading to $\mathrm{AD}$ or even aortic rupture (6). AAS occur when either a tear or an ulcer allows blood to penetrate from the aortic lumen into the media or when a rupture of the vasa vasorum causes a bleed within the media. The inflammatory response to blood in the media may lead to aortic dilatation and rupture. Figure 1 shows the DeBakey and Stanford classifications. The most common clinical features of AAS are shown in Figure $2(7,8)$.

\section{Laboratory testing}

The tests listed in Table 1 are required for differential diagnosis or the detection of complications (4). If D-Dimers are elevated the suspicion of AD is increased. Typically, the level of D-Dimers is very high $(>800 \mu \mathrm{g} / \mathrm{L})$ in the first 6 hours after the onset of symptoms compared with other disorders in which the D-Dimer level increases gradually such as pneumonia. D-Dimers yield the highest diagnostic value in the first hour (9). If the D-Dimers are negative, IMH and PAU may still be present. The advantage of the test is increased alertness in formulating a differential diagnosis.

Because AD affects the media of the aorta, several biomarkers have been developed that relate to the injury 

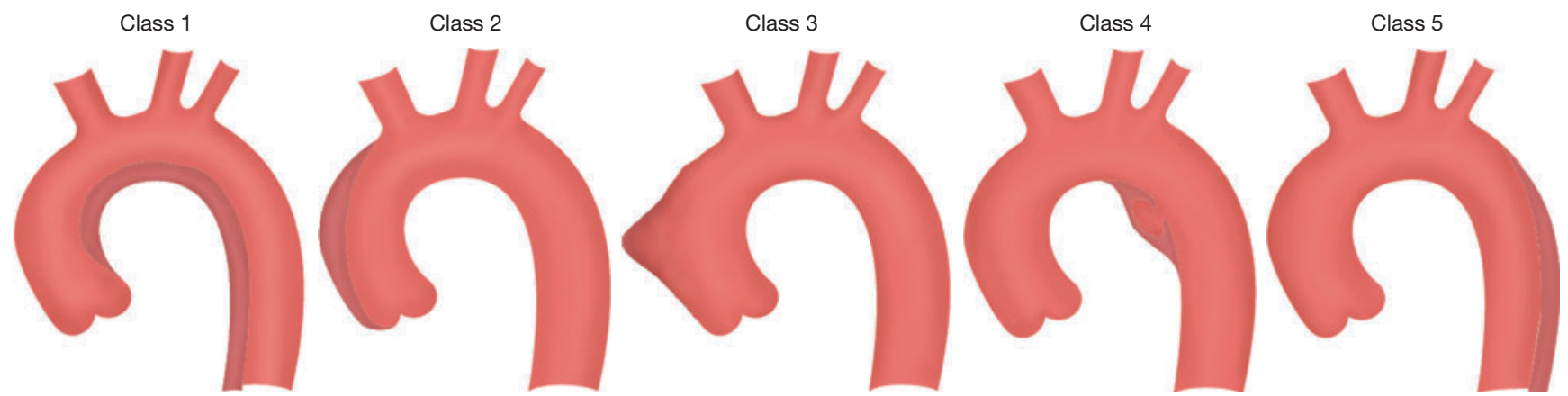

Figure 2 Classification of acute aortic syndrome in aortic dissection. Class 1: classic AD with true and false lumen with or without communication between the two lumens; class 2: intramural hematoma (IMH); class 3: subtle or discrete AD with bulging of the aortic wall; class 4: ulceration of aortic plaque following plaque rupture; class 5: iatrogenic or traumatic $\mathrm{AD}$, illustrated by a catheter-induced separation of the intima. $\mathrm{AD}$, aortic dissection.

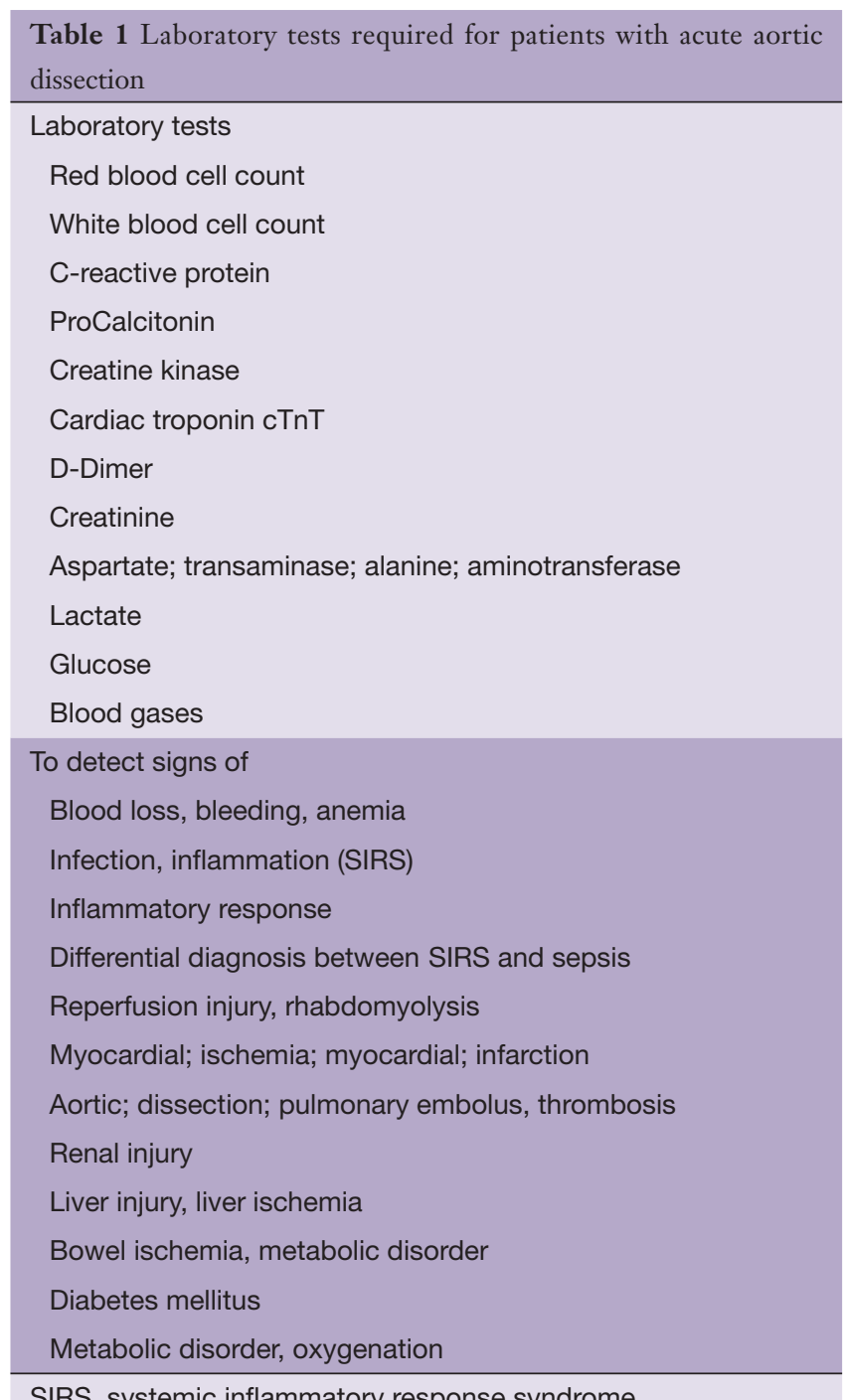

SIRS, systemic inflammatory response syndrome. of the vascular endothelial or smooth muscle cells (Smooth muscle myosin HC), the vascular interstitium (calponin, matrix metalloproteinase B), the elastic laminae (Soluble elastin fragments) or thrombosis, which are experimental at present and have not yet entered routine clinical care.

\section{Diagnostic imaging}

The main purpose of imaging is to confirm the diagnosis. But it is also vital to assess the entire aorta, including the aortic diameters, the shape and extent of the dissection membrane, the involvement of the aortic valve, aortic branches, the relationship with adjacent structures (Table 2) and the presence of mural thrombus.

Computed tomography (CT), MRI and TOE are equally reliable for the confirmation or exclusion of the diagnosis of AAD. But CT and MRI are superior to TOE for the assessment of $\mathrm{AD}$ extension and branch involvement, as well as for the diagnosis of IMH, PAU and traumatic aortic lesions. On the other hand, TOE using color flow Doppler is superior for imaging flow across tears and identifying their locations. TOE may be very helpful in the unstable patient and is used to monitor changes in the operating theatre and in postoperative intensive care.

The majority of community hospitals in Europe have CT scanning available on a 24 -hour basis and this is the commonest imaging modality in use.

\section{CT}

The key finding on contrast-enhanced images is the intimal 


\begin{tabular}{l} 
Table 2 Details required from imaging in acute aortic dissection \\
\hline Aortic dissection \\
Visualization of intimal flap \\
Extent of the disease \\
Identification of false and true lumens \\
Localisation of entry and re-entry tears \\
Identification of antegrade and/or retrograde dissection \\
Involvement of side branches \\
Detection of malperfusion \\
Detection of organ ischemia \\
Detection of pericardial effusion \\
Detection of pleural effusion \\
Detection of peri-aortic bleeding \\
Signs of mediastinal bleeding \\
Intramural hematoma \\
Localisation and extent of aortic wall thickening \\
Co-existence of atheromatous disease (calcium shift) \\
Presence of small intimal tears \\
Penetrating aortic ulcer \\
Localisation of the lesion (length and depth) \\
Coexistence of intramural hematoma \\
Involvement of the peri-aortic tissue and bleeding \\
In all cases \\
so-existence of other aortic lesions, aneurysms, plaques, \\
\hline
\end{tabular}

flap separating the two lumens. The primary role of an unenhanced acquisition is to detect medially displaced aortic calcification or the intimal flap itself. Un-enhanced images are also important for detecting IMH.

The diagnosis of $\mathrm{AD}$ can be made on transverse CT images, but multi- planar reconstruction images play an important complementary role in confirming the diagnosis and determining the extent of involvement, especially with regard to involvement of aortic branch vessels. The major role of multi- detector CT is in providing specific, precise measurements of the extent of the dissection, including length and diameter of the aorta, the true lumen (TL), the false lumen (FL), involvement of vital vasculature and the distance from the intimal tear to the vital vascular branches (10).

$\mathrm{CT}$ is the most commonly used imaging technique for $\mathrm{AD}$, because of its speed, widespread availability and excellent sensitivity of $>95 \%$ for AD. Sensitivity and specificity for diagnosing arch vessel involvement are $93 \%$ and $98 \%$ respectively with an overall accuracy of $96 \%$ (11). Triple rule out is a term that describes an ECG-gated 64-detector CT study to evaluate patients with acute chest pain in the emergency department, used for three potential causes: $\mathrm{AD}$, pulmonary embolism and coronary artery disease. Highly mobile linear intra-luminal filling defects which may mimic an intimal flap on CT, the socalled "pulsation artifact" is the most common cause of misdiagnosis (12). It is caused by pulsatile movement of the ascending aorta during the cardiac

cycle between end-diastole and end-systole. The potential problem of pulsation artifacts can be eliminated with ECG-gating or by a $180^{\circ}$ linear interpolation reconstruction algorithm.

\section{Transesophageal echocardiography (TOE)}

Intimal flaps can be detected, entry and re-entry tears localized, thrombus formation in the FL visualized and, using colour Doppler, antegrade and retrograde flow can be imaged while using pulsed or continuous wave Doppler. In addition, pressure gradients between the TL and FL can be estimated. Retrograde AD can be detected by lack of, reduced or reversed flow in the FL. Thrombus formation is often combined with slow flow and spontaneous contrast. Wide communications between the TL and FL result in extensive intimal flap movements which can lead to collapse of the TL as a mechanism of malperfusion. Localised AD of the distal segment of the ascending aorta can be missed as it corresponds with the 'blind spot' in TOE.

The sensitivity of TOE reaches $99 \%$, with a specificity of $89 \%$. The positive and negative predictive values are $89 \%$ and $99 \%$ respectively, based on surgical and/or autopsy data that were independently confirmed $(13,14)$.

\section{Magnetic resonance imaging}

MRI is considered the leading technique for the diagnosis of $\mathrm{AD}$ with a reported sensitivity and specificity of $98 \%$ (15). It demonstrates the extent of the disease and depicts the distal ascending aorta and the aortic arch in more detail than is achieved by TOE. The localization of entry and reentry is nearly as accurate as with TOE and the sensitivity of both is near to $90 \%$. The identification of the intimal flap by MRI remains the key finding. The TL shows signal void whereas the FL shows higher signal intensity, indicative of turbulent flow. 
Despite the excellent performance, MRI is less accessible than CT or TOE, especially in a critically ill patient. It is therefore most useful in acute and sub-acute Type B dissection or chronic aortic dissection.

\section{Aortography}

The angiographic diagnosis is based upon direct angiographic signs such as the visualization of the intimal flap, a negative, frequently mobile linear image or the recognition of two lumens. It may also demonstrate irregularity or compression of the aortic lumen, branch

\begin{tabular}{l} 
Table 3 Clinical data useful to assess the likelihood of acute aortic \\
syndrome \\
\hline High risk conditions \\
Positive aortic disease family history in conditions e.g., Marfan \\
syndrome \\
Aortic valve disease \\
Thoracic aortic aneurysm \\
Prior aortic intervention \\
High risk pain \\
Chest, back, abdominal \\
Abrupt onset \\
Severe intensity \\
Ripping, tearing \\
High risk examination \\
Evidence deficit of perfusion \\
Aortic diameter \\
Shock but preserved blood pressure
\end{tabular}

vessel abnormalities, thickening of the aortic walls and aortic regurgitation (13).

This technique is no longer used for the diagnosis of $\mathrm{AD}$ except during coronary arteriography or endovascular intervention.

\section{Clinical diagnosis}

In 2010 the ACC/AHA Guidelines proposed a risk assessment tool based on three groups of information: predisposing conditions, features of the pain and clinical examination and proposed a scoring system that considered the number of these groups that were involved from 1 to 3 (Table 3). IRAD reported the sensitivity of this approach, but a validation is not yet available (9). The current recommendations from the 2014 ESC Guidelines are shown in Table 4.

\section{Treatment}

Irrespective of whether the patient undergoes an intervention, medical treatment to control pain and blood pressure is essential. This is common to both ESC and AHA guidelines $(4,5)$. Where they differ is the greater emphasis in the more recent ESC document that emphasizes the growing role of endovascular treatment of thoracic aortic disease. But as yet, there are no dedicated endografts for the ascending aorta. Interventions on the ascending aorta could avoid the trauma and complexity of open surgery, especially in an ageing population with co-morbidity. It is likely that by the time of the next international guidelines some proximal aortic pathologies will be managed successfully

\begin{tabular}{|c|c|c|}
\hline Recommendation & Class & Level \\
\hline $\begin{array}{l}\text { In all patients with } A D \text {, medical therapy including pain relief } \\
\text { and blood pressure control is recommended }\end{array}$ & 1 & $\mathrm{C}$ \\
\hline In patients with Type A AD, urgent surgery is recommended & I & $\mathrm{B}$ \\
\hline $\begin{array}{l}\text { In patients with acute Type A AD and organ malperfusion, a hybrid approach } \\
\text { (i.e., ascending aorta and /or arch replacement associated with any } \\
\text { percutaneous aortic or branch artery procedure) should be considered }\end{array}$ & Ila & B \\
\hline In uncomplicated Type B AD, medical therapy should always be recommended & I & $\mathrm{C}$ \\
\hline In uncomplicated Type B AD, TEVAR should be considered & Ila & B \\
\hline In complicated Type B AD, TEVAR is recommended & I & $\mathrm{C}$ \\
\hline In uncomplicated Type B AD, surgery may be considered & $\mathrm{Ilb}$ & $\mathrm{C}$ \\
\hline
\end{tabular}


without surgery.

\section{Type A AD}

Surgery is the treatment of choice. Acute type A AD has a mortality of $50 \%$ within the first 48 hours if not operated. Despite improvements in surgical and anesthetic techniques perioperative mortality $(25 \%)$ and neurological complications $(18 \%)$ remain high $(16,17)$. But surgery reduces 1-month mortality from $90 \%$ to $30 \%$. The advantage of surgery over conservative treatment is especially obvious in the long term.

In Liverpool, UK, over the last 10 years a group of aortic surgeons have worked as a team in one hospital and established an efficient referral system for the management of $\mathrm{AD}$. One result of this activity has been to reduce the 30 -day mortality from $20 \%$ to $9.8 \%$ for AAD without being risk averse (Professor A. Oo, personal communication). In the North West Thames region of London over the last 5 years we have followed a similar pattern.

$\mathrm{AD}$ is an uncommon disease and is best treated by a dedicated aortic team who has the enthusiasm, expertise and experience to deal with these complex patients. It is generally agreed that acute $\mathrm{AD}$ is a surgical emergency and best treated in a specialized unit. The merit of transferring these unstable patients with meticulous control of pain and blood pressure remains controversial and varies among different countries depending upon the distances involved (18).

Coma, shock secondary to pericardial tamponade, malperfusion of coronary or peripheral arteries and stroke are important factors which may predict post-operative mortality. In an analysis of 936 patients with type A AD enrolled in the IRAD registry, up to the age of 80 years, inhospital mortality was significantly lower after operation than with medical treatment. In octogenarians, in-hospital mortality was lower after surgery than with medical treatment (37.9\% vs. $55.2 \%)$; but the difference failed to reach statistical significance due to the limited number of participants over the age of 80 years (19). Although some have reported excellent surgical results and quality of life outcomes in the elderly (20), others found a higher rate of post-operative neurological complications (21). Based on current evidence, age per se should not be considered an exclusion criterion for surgical treatment.

In most cases of aortic regurgitation associated with type A the aortic valve is essentially normal and can be preserved by applying an aortic valve-sparing repair. But in the context of an emergency and an unstable patient, an aortic valve replacement can be performed (22). A controversial issue is the extent of repair. Ascending aortic replacement or hemi-arch alone is technically easier and effectively closes the entry site, but leaves a large part of the diseased aorta untreated.

\section{Malperfusion}

Patients with visceral or renal malperfusion in acute type A $\mathrm{AD}$ often have their primary tear close to the descending aorta. Malperfusion syndrome occurs in up to $30 \%$ of patients with acute aortic dissection. Visceral organ and limb ischemia is caused by dynamic compression of the TL, due to high-pressure accumulation in the FL as a result of the large proximal flow into the thoracic aorta FL and insufficient outflow into the distal aorta.

In most instances malperfusion is caused by a combination of dynamic and static obstruction; therefore surgical or hybrid treatment should be considered for patients with organ malperfusion. The classic technique involves puncture of the intimal flap using a Brockenborough needle via a transfemoral approach. An alternative technique described in 2000 (23) is the so-called "scissor" method. It is based on the insertion of two stiff guide wires, one in the TL and the other in the FL through a single transfemoral sheath. But fenestration may not resolve malperfusion completely. In a recent study (24), 75\% of patients undergoing fenestration required additional endovascular interventions for relief of ischemia.

These patients may benefit from extended treatment such as "frozen elephant trunk" repair in order to close the primary entry tear and decompress the TL. The importance of intra-operative imaging and of immediate post-operative imaging, ideally in a hybrid suite, is obvious.

By contrast, more extensive repair, including graft replacement of the ascending aorta and aortic arch and integrated stent grafting of the descending aorta $(25,26)$ as a one stage procedure is technically more challenging and prolongs the operation, with an increased risk of neurological complications (27), but offers the advantage of a complete repair, with a low likelihood of re-intervention (28).

Controversy persists over whether surgery should be performed in patients with acute Type A AD who present with neurological deficits or coma. Although commonly associated with a poor post-operative prognosis, recovery has been reported when rapid brain reperfusion is achieved $(29,30)$ especially if the time between symptom onset and arrival at the operating theatre is less than 5 hours (31). 
Endovascular treatment alone to treat acute type A AD has been attempted in highly selected cases but has not been validated (32-34).

\section{Conclusions}

Aortic surgery is one of the few areas in acquired cardiac surgery which in 2016 is expanding. Although the latest 2014 guidelines are comprehensive, advice regarding the treatment of patients is at the $11 \mathrm{~B}$ or $\mathrm{C}$ level. There is increasing use of endovascular approaches for acute dissection based on extensive experience in aortic aneurysms in both abdominal and thoracic compartments. Much depends on the judgment of the individual surgeon working in conjunction with radiologists and interventional cardiologists within the environment of their unit. This has become a truly multi-disciplinary activity.

\section{Acknowledgements}

None.

\section{Footnote}

Conflicts of Interest: The author has no conflicts of interest to declare.

\section{References}

1. Olsson C, Thelin S, Ståhle E, et al. Thoracic aortic aneurysm and dissection: increasing prevalence and improved outcomes reported in a nationwide populationbased study of more than 14,000 cases from 1987 to 2002 . Circulation 2006;114:2611-8.

2. Howard DP, Banerjee A, Fairhead JF, et al. Populationbased study of incidence and outcome of acute aortic dissection and premorbid risk factor control: 10-year results from the Oxford Vascular Study. Circulation 2013;127:2031-7.

3. Landenhed M, Engström G, Gottsäter A, et al. Risk profiles for aortic dissection and ruptured or surgically treated aneurysms: a prospective cohort study. J Am Heart Assoc 2015;4:e01513.

4. Erbel R, Aboyans V, Boileau C, et al. 2014 ESC Guidelines on the diagnosis and treatment of aortic diseases: Document covering acute and chronic aortic diseases of the thoracic and abdominal aorta of the adult. The Task Force for the Diagnosis and Treatment of
Aortic Diseases of the European Society of Cardiology (ESC). Eur Heart J 2014;35:2873-926.

5. Hiratzka LF, Bakris GL, Beckman JA, et al. 2010 ACCF/AHA/AATS/ACR/ASA/SCA/SCAI/SIR/STS/ SVM guidelines for the diagnosis and management of patients with Thoracic Aortic Disease: a report of the American College of Cardiology Foundation/American Heart Association Task Force on Practice Guidelines, American Association for Thoracic Surgery, American College of Radiology, American Stroke Association, Society of Cardiovascular Anesthesiologists, Society for Cardiovascular Angiography and Interventions, Society of Interventional Radiology, Society of Thoracic Surgeons, and Society for Vascular Medicine. Circulation 2010;121:e266-369.

6. Sampson UK, Norman PE, Fowkes FG, et al. Global and regional burden of aortic dissection and aneurysms: mortality trends in 21 world regions, 1990 to 2010. Glob Heart 2014;9:171-80.

7. Erbel R, Alfonso F, Boileau C, et al. Diagnosis and management of aortic dissection. Eur Heart J 2001;22:1642-81.

8. Reul GJ, Cooley DA, Hallman GL, et al. Dissecting aneurysm of the descending aorta. Improved surgical results in 91 patients. Arch Surg 1975;110:632-40.

9. Svensson LG, Labib SB, Eisenhauer AC, et al. Intimal tear without hematoma: an important variant of aortic dissection that can elude current imaging techniques. Circulation 1999;99:1331-6.

10. Rogers AM, Hermann LK, Booher AM, et al. Sensitivity of the aortic dissection detection risk score, a novel guidelinebased tool for identification of acute aortic dissection at initial presentation: results from the international registry of acute aortic dissection. Circulation 2011;123:2213-8.

11. LePage MA, Quint LE, Sonnad SS, et al. Aortic dissection: CT features that distinguish true lumen from false lumen. AJR Am J Roentgenol 2001;177:207-11.

12. Sommer T, Fehske W, Holzknecht N, et al. Aortic dissection: a comparative study of diagnosis with spiral CT, multiplanar transesophageal echocardiography, and MR imaging. Radiology 1996;199:347-52.

13. Posniak HV, Olson MC, Demos TC. Aortic motion artifact simulating dissection on CT scans: elimination with reconstructive segmented images. AJR Am J Roentgenol 1993;161:557-8.

14. Erbel R, Engberding R, Daniel W, et al. Echocardiography in diagnosis of aortic dissection. Lancet 1989;1:457-61.

15. Guéret $P$, Sénéchal C, Roudaut R, et al. Comparison of 
transesophageal and transthoracic echocardiography in acute aortic dissection. A multi-center prospective study. J Am Coll Cardiol 1991;17:A260-A260.

16. Nienaber CA, von Kodolitsch Y, Nicolas V, et al. The diagnosis of thoracic aortic dissection by noninvasive imaging procedures. N Engl J Med 1993;328:1-9.

17. Chiappini B, Schepens M, Tan E, et al. Early and late outcomes of acute type A aortic dissection: analysis of risk factors in 487 consecutive patients. Eur Heart J 2005;26:180-6.

18. Trimarchi S, Nienaber CA, Rampoldi V, et al. Contemporary results of surgery in acute type A aortic dissection: The International Registry of Acute Aortic Dissection experience. J Thorac Cardiovasc Surg 2005;129:112-22.

19. Pape LA, Awais M, Woznicki EM, et al. Presentation, Diagnosis, and Outcomes of Acute Aortic Dissection: $17-$ Year Trends From the International Registry of Acute Aortic Dissection. J Am Coll Cardiol 2015;66:350-8.

20. Trimarchi S, Eagle KA, Nienaber CA, et al. Role of age in acute type A aortic dissection outcome: report from the International Registry of Acute Aortic Dissection (IRAD). J Thorac Cardiovasc Surg 2010;140:784-9.

21. Tang GH, Malekan R, Yu CJ, et al. Surgery for acute type A aortic dissection in octogenarians is justified. J Thorac Cardiovasc Surg 2013;145:S186-90.

22. Shrestha M, Khaladj N, Haverich A, et al. Is treatment of acute type A aortic dissection in septuagenarians justifiable? Asian Cardiovasc Thorac Ann 2008;16:33-6.

23. Bonser RS, Ranasinghe AM, Loubani M, et al. Evidence, lack of evidence, controversy, and debate in the provision and performance of the surgery of acute type A aortic dissection. J Am Coll Cardiol 2011;58:2455-74.

24. Beregi JP, Prat A, Gaxotte V, et al. Endovascular treatment for dissection of the descending aorta. Lancet 2000;356:482-3

25. Midulla M, Renaud A, Martinelli T, et al. Endovascular fenestration in aortic dissection with acute malperfusion

Cite this article as: Pepper J. Differential aspects of the disease and treatment of Thoracic Acute Aortic Dissection (TAAD) - the European experience. Ann Cardiothorac Surg 2016;5(4):360-367. doi: 10.21037/acs.2016.06.05 syndrome: immediate and late follow-up. J Thorac Cardiovasc Surg 2011;142:66-72.

26. Weigang E, Parker JA, Czerny M, et al. Should intentional endovascular stent-graft coverage of the left subclavian artery be preceded by prophylactic revascularisation? Eur J Cardiothorac Surg 2011;40:858-68.

27. Eggebrecht H, Thompson M, Rousseau H, et al. Retrograde ascending aortic dissection during or after thoracic aortic stent graft placement: insight from the European registry on endovascular aortic repair complications. Circulation 2009;120:S276-81.

28. Leontyev S, Borger MA, Etz CD, et al. Experience with the conventional and frozen elephant trunk techniques: a single-centre study. Eur J Cardiothorac Surg 2013;44:1076-82; discussion 1083.

29. Tsagakis K, Pacini D, Di Bartolomeo R, et al. Multicenter early experience with extended aortic repair in acute aortic dissection: is simultaneous descending stent grafting justified? J Thorac Cardiovasc Surg 2010;140:S116-20; discussion S142-S146.

30. Pocar M, Passolunghi D, Moneta A, et al. Recovery of severe neurological dysfunction after restoration of cerebral blood flow in acute aortic dissection. Interact Cardiovasc Thorac Surg 2010;10:839-41.

31. Roselli EE, Rafael A, Soltesz EG, et al. Simplified frozen elephant trunk repair for acute DeBakey type I dissection. J Thorac Cardiovasc Surg 2013;145:S197-201.

32. Tsukube T, Hayashi T, Kawahira T, et al. Neurological outcomes after immediate aortic repair for acute type A aortic dissection complicated by coma. Circulation 2011;124:S163-7.

33. Zimpfer D, Czerny M, Kettenbach J, et al. Treatment of acute type a dissection by percutaneous endovascular stentgraft placement. Ann Thorac Surg 2006;82:747-9.

34. Metcalfe MJ, Holt PJ, Hinchliffe RJ, et al. Fenestrated endovascular aneurysm repair: graft complexity does not predict outcome. J Endovasc Ther 2012;19:528-35. 